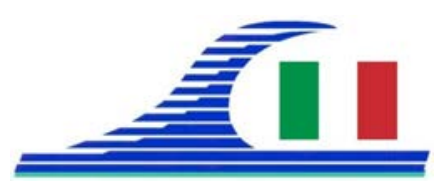

Conférence Méditerranéenne Côtière et Maritime

EDITION 3, FERRARA, ITALIA (2015)

Coastal and Maritime Mediterranean Conference

Disponible en ligne - http://www.paralia.fr - Available online

\title{
Distribution transitoire des matières en suspension dans un écoulement unidirectionnel non uniforme
}

\author{
Martin SANCHEZ ${ }^{1}$
}

\author{
1. Université de Nantes, UMR-6112 du CNRS - Planétologie et Géodynamique, \\ 2 rue de la Houssinière, BP 92208, 44322 Nantes cedex 3, France. \\ martin.sanchez@univ-nantes.fr
}

\section{Résumé :}

Une modélisation 1DH est mise en œuvre pour étudier un cas particulier de transport en suspension dans un canal correspondant à un problème 2DV. Pour inclure la dimension verticale, dans la modélisation, un modèle dénommé $\alpha-\beta$ est utilisé. Ce dernier a été développé pour décrire la distribution verticale des sédiments fins en suspension en régime non stationnaire et/ou non uniforme, incluant les phénomènes de dépôt et de remise en suspension des sédiments (SANCHEZ, 2013). Les résultats obtenus montrent que dans certains secteurs du domaine étudié, le modèle $\alpha$ - $\beta$ décrivant la distribution verticale des matières en suspension peut être simplifié.

Most-clés : Sédiments en suspension, Profil vertical de concentration, Ecoulement non uniforme, Régime transitoire, Dépôt, Erosion, Vase, Sable, Modèle $\alpha$ - $\beta$.

\section{Introduction}

Les modèles hydrodynamiques 2DH actuels permettent des applications sur des très longues durées avec une échelle géométrique fine dans les maillages. Si les variables du problème sont convenablement intégrées sur la verticale, les résultats obtenus de ces modèles se rapprochent de ceux issus des meilleures modélisations 3D.

Les applications pratiques sur des problèmes réels utilisent très largement les modèles 2DH. La dimension verticale peut être prise en compte d'une façon réaliste et précise dans plusieurs types de problème. Voici deux exemples :

a) Dans les modèles de propagation de houle décrivant la distribution verticale du potentiel de vitesses avec la théorie de Stokes-Airy (BERKHOFF, 1972).

b) Dans les modèles courantologiques qui intègrent sur toute la profondeur l'effet des contraintes de cisaillement sur les vitesses moyennées verticalement (SAINTVENANT, 1871).

Grâce à un modèle dénommé $\alpha$ - $\beta$ qui a été récemment développé (SANCHEZ, 2013), une description précise de la répartition verticale des sédiments dans la colonne d'eau est obtenue. Cela rend possible la simulation du transport sédimentaire à l'aide de modèles $2 \mathrm{DH}$ avec une prise en compte de l'aspect transitoire du régime, des mélanges verticaux convectifs-diffusifs, et des échanges solides avec le fond (érosion et dépôt des sédiments). 
Côtes méditerranéennes menacées :

Risques et défis dans le contexte du changement climatique

Les premières validations du modèle $\alpha$ - $\beta$ ont été effectuées en régime non stationnaire pour des vitesses de chute $W$ faibles $\left(0,05<W\left(\mathrm{~mm} \mathrm{~s}^{-1}\right)<3,2\right)$ caractéristiques des sédiments fins (SANCHEZ, 2013). Par la suite ce modèle a été utilisé avec succès pour décrire le transport en suspension de sables et graviers sous actions hydrodynamiques cycliques dans une large gamme de période $T(2,5<T(\mathrm{~s})<\infty)$, (SANCHEZ, 2014).

L'objet de cet article est d'étudier, en régime non-uniforme, le transport en suspension en canal. La configuration examinée peut représenter, soit un cours d'eau arrivant à un lac ou à une retenue de barrage, soit un estuaire débouchant sur une mer microtidale.

\section{Théorie en régime établi}

Si le régime sédimentaire est uniforme et stationnaire, la distribution verticale de la concentration $C$ en matières en suspension est régie par :

$W C=-K_{z} \frac{\partial C}{\partial z}$

où $W$ est la valeur locale de la vitesse de chute des matières en suspension, $z$ la coordonnée verticale et $K_{z}$ le coefficient de diffusion turbulente de masse selon la direction $\mathrm{Oz}$. Dans les équations qui suivent on considère que le fond se situe en $z=0$.

Dans cette étude, les grandeurs $W$ et $K_{z}$ sont considérées être indépendantes de $z$. Cette hypothèse est une simplification du problème très utilisée dans les applications pratiques. Ainsi, en régime établi l'expression de la concentration s'écrit :

$\dddot{C}\left(z^{\circ}\right)=C(0) \exp \left(-\alpha_{\infty} z^{\circ}\right)$

où $z^{\circ}=z / d$ est la coordonnée verticale adimensionnelle, $d$ la profondeur et $\alpha_{\infty}$ le nombre de Peclet caractéristique des transferts verticaux de sédiments par convection-diffusion : $\alpha_{\infty}=\frac{W d}{K_{Z}}$

Une relation usuelle pour évaluer $K_{\mathrm{z}}$ qui est retenue dans cette étude est la suivante :

$K_{z}=\frac{\kappa}{6} U_{c} d$

où $U_{c}$ est la vitesse de cisaillement et $\kappa \approx 0,4$ la constante universelle de Karman.

\section{Présentation du modèle $\alpha-\beta$ (SANCHEZ, 2013)}

Des tests numériques effectués pendant la phase de validation du modèle $\alpha$ - $\beta$ en considérant une absence des phénomènes d'érosion et de dépôt, ont montré qu'avec des conditions hydrodynamiques comportant des accélérations, la concentration $C$ n'est plus décrite par l'équation 2. Selon le modèle $\alpha$ - $\beta$, dans ce cas de régime transitoire, le profil de la concentration noté $\widetilde{C}$ est correctement modélisé par l'équation suivante :

$\tilde{C}\left(z^{\circ}\right)=C(0) \exp \left(-\alpha z^{\circ}\right)$ où $\alpha$ est un paramètre dont la valeur tend toujours vers $\alpha_{\infty}$ selon une loi phénoménologique qui pour un écoulement unidimensionnel 1DH (direction $O x$ ), s'écrit : 
$\frac{\partial \alpha}{\partial t}+\bar{V}_{x} \frac{\partial \alpha}{\partial x}=c_{\alpha} \frac{U_{c}}{d}\left(\alpha_{\infty}-\alpha\right)$

où $\bar{V}_{x}$ est la vitesse moyenne sur la verticale et $c_{\alpha} \approx 0,667$ est le coefficient du modèle.

Dans la plupart des cas, le dépôt et l'érosion des sédiments modifient de façon plus significative le profil vertical de la concentration $C$ que le caractère transitoire des conditions hydrodynamiques. Il est montré que dans tous les cas le profil de $C$ est bien décrit par (SANCHEZ, 2013) :

$C\left(z^{\circ}\right)=C_{R} \exp \left(-\alpha z^{\circ}\right) \exp \left(-\beta\left(1-z^{\circ}\right)^{2}\right)$

où $C_{R}$ est une concentration de référence et $\beta$ un paramètre dont la valeur tend toujours vers $\beta_{\infty}$ selon la loi phénoménologique ci-dessous, incluant un coefficient $c_{\beta}$ :

$\frac{\partial \beta}{\partial t}+\bar{V}_{x} \frac{\partial \beta}{\partial x}=c_{\beta} \frac{U_{c}}{d}\left(\beta_{\infty}-\beta\right)$

où $\beta_{\infty}$ est la valeur terminale instantanée du paramètre $\beta$, qui dépend des échanges solides entre le fond et la colonne d'eau, échanges paramétrés par le taux de dépôt $D^{e f}$ (terme puits en $\mathrm{kg} \mathrm{m}^{-2} \mathrm{~s}^{-1}$ ) ou par le taux d'érosion $E^{\text {ef }}$ (terme source en $\mathrm{kg} \mathrm{m}^{-2} \mathrm{~s}^{-1}$ ).

Dans ce qui suit on utilise un seul taux d'échanges solides avec le fond $S^{\text {ef }}$ qui est égal à $D^{e f}$ en cas de dépôt et égal à $-E^{e f}$ en cas d'érosion. De même, on généralise une loi de KRONE (1986) afin de définir un taux adimensionnel d'échanges solides avec le fond :

$p=\frac{S^{e f}}{W \times C(0)}$

Il est montré (SANCHEZ, 2013) que si le paramètre $p$ et les conditions hydrodynamiques restent constantes dans le temps, $\alpha$ converge vers $\alpha_{\infty}$ et $\beta$ vers $\beta_{\infty}$. Dans ce cas les conditions de frontière liées aux échanges solides avec le fond sont satisfaites si :

$\beta_{\infty}=0,50 p \alpha$

En complément, en cas d'érosion ou d'échanges solides nuls avec le fond $(p \leq 0)$ on a :

$c_{\beta}=0,667$

Finalement, en cas de dépôt $(p>0)$ on a :

$c_{\beta}=0,667+0,3 \times \beta_{\infty}$

\section{Méthodes}

Le modèle $\alpha$ - $\beta$ en version $1 \mathrm{DH}$ est appliqué à l'étude du transport de sédiments en suspension par un écoulement 2DV unidirectionnel (sens du courant Ox). La configuration imposée du canal étudié est montrée en haut de la figure 1. Ce canal comporte une région d'érosion entre $\mathrm{A}$ et $\mathrm{B}$, une région de dépôt en aval de $\mathrm{C}$, et entre ces deux régions (entre $B$ et $C$ ) un secteur sans érosion ni dépôt. Dans les simulations, la profondeur $d=2 \mathrm{~m}$ et la vitesse de chute $W=0,001 \mathrm{~m} \mathrm{~s}^{-1}$ sont maintenues constantes. De plus, les phénomènes hydrodynamiques transitoires au niveau des discontinuités sont négligés. Le tableau 1 rassemble les principaux paramètres du problème étudié. 
Côtes méditerranéennes menacées :

Risques et défis dans le contexte du changement climatique

Tableau 1. Récapitulatif des paramètres hydrosédimentaires du problème étudié.

\begin{tabular}{llllll}
\hline & En amont de A & Entre A et B & Entre B et C & Entre C et D & En aval de D \\
\hline Vitesse du courant $\bar{V}_{X}\left(\mathrm{~m} \mathrm{~s}^{-1}\right)$ & 1,28 & 1,28 & 0,64 & 0,32 & 0,32 \\
Vitesse de cisaillement $U_{c}\left(\mathrm{~m} \mathrm{~s}^{-1}\right)$ & 0,08 & 0,08 & 0,04 & 0,02 & 0,02 \\
Longueur $(m)$ & indéfinie & 760 & 760 & 200 & indéfinie \\
Taux d'échanges solides $(p)$ & 0 & -1 & 0 & +1 & +1 \\
\hline
\end{tabular}
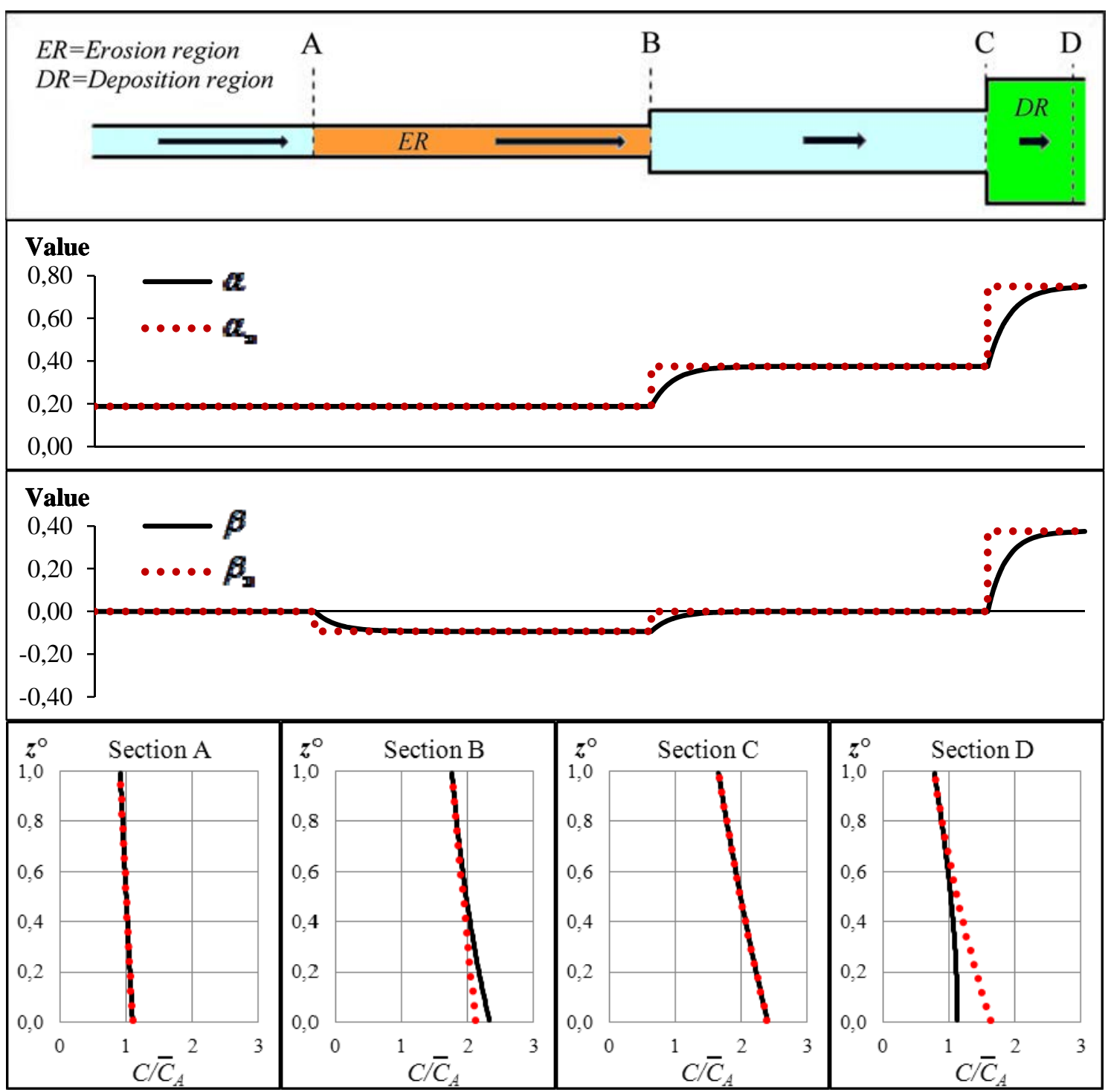

Figure 1. Présentation des résultats obtenus par l'application du modèle $\alpha-\beta$.

En haut : Schématisation de l'écoulement en canal étudié.

Au $2^{\text {ème }}$ rang : Evolution de $\alpha$ et $\alpha_{\infty}$, paramètres liés au mélange convectif-diffusif.

Au $3^{\text {ème }}$ rang : Evolution de $\beta$ et $\beta_{\infty}$, paramètres reliés aux échanges avec le fond.

En bas : Concentrations réduites par la concentration moyenne en A en abscisses, en fonction de $z^{\circ}$ en ordonnées. Les résultats du modèle $\alpha$ - $\beta$ sont tracés en trait noir et la loi exponentielle avec alpha comme seul paramètre, est tracée en pointillés rouges. 
Mediterranean coasts at threat:

Hazards and challenges in the context of climate variability

\section{Discussion des résultats obtenus}

Les résultats obtenus pour la distribution verticale de la concentration sont montrés pour 4 sections transversales $\mathrm{A}, \mathrm{B}, \mathrm{C}$ et $\mathrm{D}$ (figure 1 , en bas).

Le profil de la section $A$ représente la condition de frontière du problème avec une valeur moyenne de $C / \bar{C}_{A}$ égale à 1,00 (où $\bar{C}_{A}$ représente la concentration de référence en amont de la section $\mathrm{A}$ ), avec les résultats du modèle $\alpha$ - $\beta$ (trait noir) en accord avec la loi définie par l'équation 2 pour un régime établi (pointillés rouges).

En raison de l'érosion simulée entre $\mathrm{A}$ et $\mathrm{B}$, la valeur moyenne en $\mathrm{B}$ de $C / \bar{C}_{A}$ vaut environ 2,00. On observe que la concentration au fond selon le modèle $\alpha$ - $\beta$ est supérieure à celle prédite par la loi pour un régime établi et ceci s’explique par un apport de sédiments par le fond suite aux remises en suspension. On vérifie que la valeur de $\partial C / \partial z$ en $z=0$ est en conformité avec la condition de frontière pour une érosion paramétrée avec $p=-1$.

Entre les sections $B$ et $C$ on impose un transport en suspension sans érosion ni dépôt, de sorte que la valeur moyenne de $C / \bar{C}_{A}$ reste constante. On passe progressivement d'un profil transitoire en $\mathrm{B}$ à un profil établi en $\mathrm{C}$. En B la vitesse de cisaillement passe de $0,08 \mathrm{~m} \mathrm{~s}^{-1}$ à $0,04 \mathrm{~m} \mathrm{~s}^{-1}$, et ceci engendre une redistribution des matières en suspension entre $\mathrm{B}$ et $\mathrm{C}$ faisant croître vers l'aval l'accumulation des sédiments près du fond.

En aval de $C$ un dépôt non entravé en chute libre est modélisé faisant décroitre progressivement la quantité des matières en suspension. La valeur moyenne de $C / \bar{C}_{A}$ redevient égale à 1,00 dans la section $\mathrm{D}$, où l'on vérifie que le profil vertical de la concentration selon le modèle $\alpha-\beta$ avec $\partial C / \partial z=0$ en $z=0$, est compatible avec la condition de frontière pour un dépôt non entravé paramétré avec $p=+1$.

$\mathrm{Au} 2^{\text {ème }}$ rang, la figure 1 montre l'évolution des paramètres $\alpha$ et $\alpha_{\infty}$ caractéristiques des transferts de sédiment verticaux convectifs-diffusifs. On constate que suivant l'écoulement, le paramètre du modèle $\alpha$ converge toujours vers une valeur terminale $\alpha_{\infty}$. De leur côté, les variations de $\alpha_{\infty}$ à proximité des discontinuités de l'écoulement sont associées à un régime hydrodynamique localement non uniforme.

Selon la loi phénoménologique de variation de $\alpha$ (Eq. 6) si les variables indépendantes du problème $\left(U_{c}, W\right.$ et $\left.d\right)$ restent constantes dans le temps et le long de l'écoulement, la durée nécessaire pour parvenir à une distribution des matières en suspension en régime établi à partir d'une condition initiale arbitraire, peut être caractérisée par une constante de temps $\tau_{\alpha}=d /\left(c_{\alpha} \times U_{c}\right)$, si bien qu'après un temps $t=4,6 \times \tau_{\alpha}$ on aura : $\left(\alpha_{\infty}-\alpha\right)=\left(\alpha_{\infty}-\alpha_{\text {init }}\right) / 100$, où $\alpha_{\text {init }}$ désigne la valeur initiale de ce paramètre (à $\left.t=0\right)$.

Dans le cas d'un écoulement stationnaire non-uniforme comme celui étudié dans cet article, le parcours $\Lambda_{\alpha}$ associé à un temps $t=4,6 \times \tau_{\alpha}$ est :

$\Lambda_{\alpha}=\frac{4,6 \times d \times \bar{V}_{x}}{c_{\alpha} \times U_{c}}$

Enfin, au $3^{\text {ème }}$ rang, la figure 1 montre l'évolution de $\beta$ et $\beta_{\infty}$, paramètres reliés aux échanges solides avec le fond. On observe que le paramètre du modèle $\beta$ converge 
Côtes méditerranéennes menacées :

Risques et défis dans le contexte du changement climatique

toujours vers une valeur terminale $\beta_{\infty}$. Les variations de $\beta_{\infty}$ sont directement liées aux variations du paramètre $p$ caractérisant ces échanges solides. Avec érosion ou dépôt, à partir d'une condition initiale arbitraire caractérisée par $\beta=\beta_{\text {init à } t=0 \text {, le parcours }}$ $\Lambda_{\beta}$ nécessaire pour observer une valeur de $\beta$ telle que $\left(\beta_{\infty}-\beta\right)=\left(\beta_{\infty}-\beta_{\text {init }}\right) / 100$, est :

$\Lambda_{\beta}=\frac{4,6 \times d \times \bar{V}_{x}}{c_{\beta} \times U_{c}}$

A titre d'exemple nous pouvons citer un cas particulier avec $c_{\alpha}=c_{\beta}=0,667$ et $\bar{V}_{x} / U_{c}=16$ pour lequel on obtient $\Lambda_{\alpha}=\Lambda_{\beta}=110 \times d$.

\section{Conclusion}

La simulation numérique effectuée avec le modèle $\alpha$ - $\beta$ pour un cas d'un écoulement stationnaire non uniforme a permis de vérifier que les conditions de frontière au fond liées aux échanges solides (érosion et dépôt) sont correctement décrites par l’utilisation de l'équation 7.

Si le paramètre $p$ caractéristique des échanges solides avec le fond et les variables indépendantes du problème $\left(U_{c}, W\right.$ et $\left.d\right)$, varient graduellement le long de l'écoulement, on peut décrire correctement la distribution verticale des matières en suspension avec les simplifications suivantes : $\alpha \approx \alpha_{\infty}$ et $\beta \approx \beta_{\infty}$.

Plus précisément, si les variations $\Delta \alpha_{\infty}$ du paramètre $\alpha_{\infty}$ sur un parcours $\Lambda_{\alpha}$ le long de l'écoulement sont partout telles que $\operatorname{Abs}\left(\Delta \alpha_{\infty} / \alpha_{\infty}\right)<<1$, on peut retenir $\alpha \approx \alpha_{\infty}$.

De même, si les variations $\Delta \beta_{\infty}$ du paramètre $\beta_{\infty}$ sur une longueur $\Lambda_{\beta}$ le long de l'écoulement sont partout telles que $\operatorname{Abs}\left(\Delta \beta_{\infty} / \beta_{\infty}\right)<<1$, on peut retenir $\beta \approx \beta_{\infty}$.

On doit noter que les expressions de $\Lambda_{\alpha}$ et de $\Lambda_{\beta}$ (équations 13 et 14 , respectivement) sont issues de cette étude.

\section{Références bibliographiques}

BERKHOFF J.C.W. (1972). Computation of combined refraction-diffraction. $13^{\text {th }}$ Coastal Engineering Proceedings, ASCE ,Vancouver, Canada,, pp 471-490.

KRONE R.B. (1986). The significance of aggregate properties to transport processes. In A.J. Mehta (Ed.), Estuarine cohesive sediment dynamics, Coastal and estuarine studies n 14, Springer, Berlin, pp 66-84. http://dx.doi.org/10.1007/978-1-4612-4936-8_4

SAINT-VENANT A. (1871). Théorie du mouvement non permanent des eaux, avec application aux crues des rivières et a l'introduction de marées dans leurs lits. Comptes Rendus de l'Académie des Sciences, Tome 73, Paris, pp 147-154 \& 237-240.

SANCHEZ M. (2013). Description non stationnaire de la distribution verticale des sédiments transportés en suspension par les écoulements à surface libre, en présence de dépôt et d'érosion. Revue Paralia, Vol. 6, pp 9.1-9.30. http://dx.doi.org/10.5150/revue-paralia.2013.009 SANCHEZ M. (2014). Distribution non stationnaire des matières en suspension dans une couche limite oscillant à haute fréquence. XIII ${ }^{\text {ème }}$ Journées Nationales Génie Côtier - Génie Civil, Dunkerque, pp 495-504. http://dx.doi.org/10.5150/jngcgc.2014.054 